\title{
THE FRAMINGHAM RISK SCORE IN PATIENTS WITH PSORIASIS AND PSORIATIC ARTHRITIS
}

\author{
Sueli Carneiro ${ }^{1, *}$, Mara Diane Lisboa Tavares ${ }^{1}$, Patrícia Gusmão Acioly ${ }^{1}$, Cláudia Camargo ${ }^{1}$, Maria Alice Penetra ${ }^{1}$, Virginia \\ Januário ${ }^{1}$, Marcia Ramos-e-Silva ${ }^{1}$
}

1.Universidade Federal do Rio de Janeiro, Rio de Janeiro (RJ), Brazil.

*Corresponding author: sueli@hucff.ufrj.br

\section{BACKGROUND}

Moderate to severe psoriasis has been associated with increased cardiovascular mortality due to the higher prevalence of traditional cardiovascular risk (CVR) factors including atherosclerosis and systemic inflammation. The Framingham risk score (FRS) has been used to assess long-term risk for cardiovascular disease, which may have practical implications for the treatment and prevention of such CVR factors. The objectives were to evaluate the prevalence of CVR factors, stratifying them by the FRS in patients with moderate to severe psoriasis or psoriatic arthritis, comparing to controls matched for age and gender without inflammatory cutaneous or systemic disease.

\section{METHODS}

A cross-sectional study was performed in three groups of patients: group 1 (41 PsO), group 2 (50 PsA) psoriatic and group 3 (40 controls). Data on clinical history of all participants such as age, gender, current smoking, use of blood pressure and/or diabetes medications, laboratory parameters such as serum total cholesterol and HDL cholesterol, glucose levels in the years of 2019-2020. The data were analyzed and applied to an FRS stratification (high $>20$, intermediate 10 to 20 , low $<10$ ). A prevalence analysis of CVR was performed.

\section{RESULTS}

Out of 85 psoriatic patients, 45\% had high CVR. In the PsO group, the CVR was high, intermediate and low in 45, 24 and $31 \%$, respectively. In the PsA group, the risk was high, intermediate and low in 46, 30 and $24 \%$, respectively, while in control group CVR was 34,25 and $41 \%$, in-particular.

\section{CONCLUSION}

The high CVR was more frequent in the PsA group followed by the PsO group and the last one was the control group. The high CVR was $12 \%$ greater in the PsA group than in the control group and 1\% higher than $\mathrm{PsO}$ group. Considering the two groups of psoriatic patients a high CVR was $11 \%$ greater than the control group. Estimating CVR is a difficult task and a major challenge in preventing cardiovascular events in the general population and this challenge is much greater in psoriasis disease, which is a systemic inflammatory disease. Therefore, CVR assessment should be the goal of holistic treatment of patients with psoriatic disease in an attempt to prevent and treat cardiovascular events.

\section{KEYWORDS}

Heart disease risk factors, Spondyloarthritis, Arthritis, Psoriasis. 